\title{
Coloring graphs from random lists of fixed size
}

\author{
Carl Johan Casselgren
}

\section{Linköping University Post Print}

\section{Tweet}

N.B.: When citing this work, cite the original article.

Original Publication:

Carl Johan Casselgren, Coloring graphs from random lists of fixed size, 2014, Random structures \& algorithms (Print), (44), 3, 317-327.

http://dx.doi.org/10.1002/rsa.20469

Copyright: Wiley: 12 months

http://eu.wiley.com/WileyCDA/

Postprint available at: Linköping University Electronic Press

http://urn.kb.se/resolve?urn=urn:nbn:se:liu:diva-107123 


\title{
Coloring graphs from random lists of fixed size
}

\author{
Carl Johan Casselgren* \\ Department of Mathematics, Umeå University, \\ SE-901 87 Umeå, Sweden
}

\begin{abstract}
Let $G=G(n)$ be a graph on $n$ vertices with maximum degree bounded by some absolute constant $\Delta$. Assign to each vertex $v$ of $G$ a list $L(v)$ of colors by choosing each list uniformly at random from all $k$-subsets of a color set $\mathcal{C}$ of size $\sigma(n)$. Such a list assignment is called a random $(k, \mathcal{C})$-list assignment. In this paper, we are interested in determining the asymptotic probability (as $n \rightarrow \infty$ ) of the existence of a proper coloring $\varphi$ of $G$, such that $\varphi(v) \in L(v)$ for every vertex $v$ of $G$. We show, for all fixed $k$ and growing $n$, that if $\sigma(n)=\omega\left(n^{1 / k^{2}}\right)$, then the probability that $G$ has such a proper coloring tends to 1 as $n \rightarrow \infty$. A similar result for complete graphs is also obtained: if $\sigma(n) \geq 1.223 n$ and $L$ is a random $(3, \mathcal{C})$-list assignment for the complete graph $K_{n}$ on $n$ vertices, then the probability that $K_{n}$ has an $L$-coloring tends to 1 as $n \rightarrow \infty$.
\end{abstract}

Keywords: list coloring, random list

\section{Introduction}

Given a graph $G$, assign to each vertex $v$ of $G$ a set $L(v)$ of colors (positive integers). Such an assignment $L$ is called a list assignment for $G$ and the sets $L(v)$ are referred to as lists or color lists. If all lists have equal size $k$, then $L$ is called a $k$-list assignment. We then want to find a proper vertex coloring $\varphi$ of $G$, such that $\varphi(v) \in L(v)$ for all $v \in V(G)$. If such a coloring $\varphi$ exists then $G$ is $L$-colorable and $\varphi$ is called an $L$-coloring. Furthermore, $G$ is called $k$-choosable if it is $L$-colorable for every $k$-list assignment $L$.

This particular variant of vertex coloring is known as list coloring and was introduced by Vizing [10] and independently by Erdös et al. [5].

Consider now the following problem: Assign lists of colors to the vertices of a graph $G=G(n)$ with $n$ vertices by choosing for each vertex $v$ its list $L(v)$ independently and uniformly at random from all $k$-subsets of a color set $\mathcal{C}=\{1,2, \ldots, \sigma\}$. Such a list assignment is called a random $(k, \mathcal{C})$ list assignment for $G$. Intuitively it should hold that the larger $\sigma$ is, the more spread are the colors chosen for the lists and thus the more likely it is that we can find a proper coloring of $G$ with colors from the lists. The question that we address in this paper is how large $\sigma=\sigma(n)$ should be in order to guarantee that with high probability ${ }^{1}$ (as $n \rightarrow \infty$ ) there is a proper coloring of the vertices of $G$ with colors from the random list assignment.

${ }^{*}$ E-mail address: carl-johan.casselgren@math.umu.se

${ }^{1}$ An event $A_{n}$ occurs with high probability if $\lim _{n \rightarrow \infty} \mathbb{P}\left[A_{n}\right]=1$. 
This problem was first studied by Krivelevich and Nachmias $[7,8]$ for the case of powers of cycles and the case of a complete bipartite graph where the parts have equal size $n$. In the latter case they showed that for all fixed $k \geq 2$, the property of having a proper coloring from a random $(k, \mathcal{C})$-list assignment exhibits a sharp threshold, and that the location of that threshold is exactly $\sigma(n)=2 n$ for $k=2$. In [3], we generalized the second part of this result and showed that for a complete multipartite graph with $s$ parts (fixed $s \geq 3$ ) of equal size $n$, the property of having a proper coloring from a random $(2, \mathcal{C})$-list assignment, has a sharp threshold at $\sigma(n)=2(s-1) n$.

Let $C_{n}^{r}$ be the $r$ th power of a cycle with $n$ vertices. For powers of cycles, Krivelevich and Nachmias proved the following theorem.

Theorem 1.1. [7] Assume $r, k$ are fixed integers satisfying $r \geq k$ and let $L$ be a random $(k, \mathcal{C})$-list assignment for $C_{n}^{r}$. If we denote by $p_{C}(n)$ the probability that $C_{n}^{r}$ is L-colorable, then

$$
p_{C}(n)= \begin{cases}o(1), & \sigma(n)=o\left(n^{1 / k^{2}}\right) \\ 1-o(1), & \sigma(n)=\omega\left(n^{1 / k^{2}}\right)\end{cases}
$$

In [4] we generalized Theorem 1.1 for the case of random $(2, \mathcal{C})$-list assignments. The following theorem is the main result of [4].

Theorem 1.2. [4] Let $g$ be a fixed odd positive integer and let $G=G(n)$ be a graph on $n$ vertices with girth $g$ and maximum degree bounded by some absolute constant. Suppose that $L$ is a random $(2, \mathcal{C})$-list assignment for $G$. If $\sigma(n)=\omega\left(n^{1 /(2 g-2)}\right)$, then the probability that $G$ has an L-coloring tends to 1 as $n \rightarrow \infty$.

Note that Theorem 1.2 implies that $\sigma(n)=\omega\left(n^{1 / 4}\right)$ colors suffices for all asymptotic families of graphs with bounded maximum degree. In [4] it is also shown that Theorem 1.2 is best possible in the sense that for each fixed odd integer $g$ and each integer $n \geq g$ there is a graph $H=H(n, g)$ with $n$ vertices, bounded maximum degree and girth $g$, such that if $\sigma(n)=o\left(n^{1 /(2 g-2)}\right)$, then the probability that $H$ has a proper coloring from a random $(2, \mathcal{C})$-list assignment tends to 0 as $n \rightarrow \infty$. Corresponding results are derived for graphs with even girth.

In this paper we consider random $(k, \mathcal{C})$-list assignments for fixed $k \geq 3$. The main result of this paper is the following theorem.

Theorem 1.3. Let $G=G(n)$ be a graph on $n$ vertices with maximum degree bounded by some absolute constant, $k \geq 3$ a fixed positive integer, and $L$ a random $(k, \mathcal{C})$-list assignment for $G$. If $\sigma(n)=\omega\left(n^{1 / k^{2}}\right)$, then the probability that $G$ has an L-coloring tends to 1 as $n \rightarrow \infty$.

Note that Theorem 1.3 generalizes Theorem 1.1. Additionally, by Theorem 1.1 it is best possible. Furthermore, it is easy to verify that Theorem 1.3 holds (and is best possible) for the case when $k=1$. Hence, Theorem 1.3 holds for any integer $k \geq 1$.

Next, we consider the corresponding problem for a complete graph $K_{n}$ on $n$ vertices. In [2] we proved that for a complete graph on $n$ vertices, the property of being colorable from a random $(1, \mathcal{C})$-list assignment has a coarse threshold at $\sigma(n)=n^{2}$. Moreover, for $K_{n}$ the property of being colorable from a random $(2, \mathcal{C})$-list assignment has a sharp threshold at $\sigma(n)=2 n$ [4]. In this paper we give a lower bound on the size of $\mathcal{C}$ which ensures that with high probability $K_{n}$ has a proper coloring from a random $(k, \mathcal{C})$-list assigment when $k \geq 3$.

Theorem 1.4. Let $L$ be a random $(3, \mathcal{C})$-list assignment for $K_{n}$. There is a number $s^{*} \approx 1.222$ such that for any $s>s^{*}$, if $\sigma(n) \geq s n$, then the probability that $K_{n}$ has an L-coloring tends to 1 as $n \rightarrow \infty$. 
Corollary 1.5. Let $L$ be a random $(k, \mathcal{C})$-list assignment for $K_{n}$, where $k \geq 3$. If $\sigma(n) \geq 1.223 n$, then the probability that $K_{n}$ has an L-coloring tends to 1 as $n \rightarrow \infty$.

Throughout the paper, our asymptotic notation and assumptions are standard. In particular, we assume that the parameter $n$ is large enough whenever necessary.

\section{Graphs with bounded maximum degree}

In this section we prove Theorem 1.3. Let $H$ be a graph and $L$ a list assignment for $H$. If $H$ is not $L$-colorable, but removing any vertex from $H$ yields an $L$-colorable graph, then $H$ is $L$-vertex-critical (or just $L$-critical). Obviously, if $L$ is a list assignment for a graph $G$, and $G$ is not $L$-colorable, then it contains a connected induced $L$-critical subgraph.

Suppose now that $H-w_{1}$ is $L$-colorable, where $w_{1}$ is some vertex of $H$. Given an $L$-coloring $\varphi$ of $H-w_{1}$, a path $P=w_{1} w_{2} \ldots w_{t}$ in $H$ is called $(\varphi, L)$-alternating if there are colors $c_{2}, c_{3}, \ldots, c_{t}$ such that $\varphi\left(w_{i}\right)=c_{i}$ and $c_{i} \in L\left(w_{i-1}\right), i=2, \ldots, t$. We allow such a path to have length 0 and thus only consist of $w_{1}$. The set of vertices which are adjacent to a vertex $x$ in a graph $G$ is denoted by $N_{G}(x)$.

Lemma 2.1. Let $H$ be a graph and $L$ a list assignment for $H$. If $H$ is L-critical, then for any vertex $v_{1} \in V(H), H-v_{1}$ has an $L$-coloring $\varphi$ that satisfies the following conditions:

(i) all vertices in $H$ lie on $(\varphi, L)$-alternating paths with origin at $v_{1}$;

(ii) for each color $c \in L\left(v_{1}\right)$, there is a vertex $w \in N_{H}\left(v_{1}\right)$, such that $\varphi(w)=c$.

(iii) Define a rank function $R: V(H) \rightarrow\{1,2, \ldots,|V(H)|\}$ on the vertices of $H$ by setting $R(u)=j$ if a shortest $(\varphi, L)$-alternating path from $v_{1}$ to $u$ has length $j$. Then for every vertex $x$ of $H-v_{1}$ and every color $c \in L(x) \backslash\{\varphi(x)\}$, there is either

(a) a vertex $y \in N_{H}(x)$ colored $\varphi(y)=c$ or

(b) a vertex $z \in N_{H}(x)$ such that $c \in L(z)$ and $R(z)<R(x)$.

Proof. Let $v_{1}$ be any vertex of $H$. Since $H$ is $L$-critical, $H-v_{1}$ is $L$-colorable. For an $L$-coloring $\psi$ of $H-v_{1}$, let $W^{\psi}$ be the set of vertices $w$ for which there is a $(\psi, L)$-alternating path from $v_{1}$ to $w$. Next, let $W_{i}^{\psi}$ be the subset of $W^{\psi}$ of vertices $w$ such that a shortest $(\psi, L)$-alternating path from $v_{1}$ to $w$ has length $i$. Let $d$ be the maximum integer for which there is an $L$-coloring $\gamma$ of $H-v_{1}$ such that $W_{d}^{\gamma}$ is non-empty.

We define $\theta_{1}$ to be the set of $L$-colorings $\psi$ of $H-v_{1}$ with $\left|W_{1}^{\psi}\right|$ minimum, that is, $\theta_{1}$ is the set of $L$-colorings $\psi$ such that there is no $L$-coloring $\psi^{\prime}$ of $H-v_{1}$ with $\left|W_{1}^{\psi^{\prime}}\right|<\left|W_{1}^{\psi}\right|$. Similarly, for each $i=2, \ldots, d$, let $\theta_{i} \subseteq \theta_{i-1}$ be the subset of $\theta_{i-1}$ consisting of $L$-colorings $\psi$ with $\left|W_{i}^{\psi}\right|$ minimum, that is, $\theta_{i}$ is the set of colorings $\psi \in \theta_{i-1}$ such that there is no $L$-coloring $\psi^{\prime} \in \theta_{i-1}$ satisfying $\left|W_{i}^{\psi^{\prime}}\right|<\left|W_{i}^{\psi}\right|$. By definition, $\theta_{i}$ is non-empty for all $i=1, \ldots, d$. Let $\varphi \in \theta_{d}$ and set $F=H\left[W^{\varphi}\right]$.

Suppose that $F \neq H$, which means that $V(H) \backslash V(F) \neq \emptyset$. Let $u \in V(H) \backslash V(F)$ and note that the color $\varphi(u)$ does not appear in any list of vertices in $F$ that are adjacent to $u$ in $H$. (This follows from the fact that $F$ contains all vertices that belong to $(\varphi, L)$-alternating paths with origin at $\left.v_{1}\right)$. Also note that the restriction of $\varphi$ to $V(H) \backslash V(F)$ is an $L$-coloring of $H-V(F)$. Next, let $\alpha$ be an $L$-coloring of $F$. Such a coloring $\alpha$ exists because $H$ is $L$-critical. Since for every vertex $u \in V(H) \backslash V(F)$, the color $\varphi(u)$ does not appear in lists of vertices in $N_{H}(u) \cap V(F)$, the restriction of $\varphi$ to $V(H) \backslash V(F)$ and $\alpha$ together make up an $L$-coloring of $H$. This contradicts the fact that $H$ is $L$-critical, and thus $F=H$. By the construction of $F$, (i) holds. 
Next, note that (ii) holds, since suppose that there is some color $c \in L\left(v_{1}\right)$, such that no neighbor of $v_{1}$ in $H$ is colored $c$. This means that we can extend $\varphi$ to a (proper) $L$-coloring of $H$, which contradicts that $H$ is not $L$-colorable. Hence, (ii) is true.

Consider now the rank function $R$ defined above. We show that (a) or (b) of condition (iii) holds for every vertex $u$ of $H-v_{1}$ and for every color of $L(u) \backslash\{\varphi(u)\}$. Suppose that $x \in W_{l}^{\varphi}$, where $l \geq 1$, and that there is some color $c \in L(x)$ such that neither (a) nor (b) holds for $x$ and c. Then no neighbor of $x$ in $H$ is colored $c$. Additionally, there is no vertex $z \in N_{H}(x)$, such that $R(z)<R(x)$ and $c \in L(z)$. Define a new coloring $\varphi^{\prime}$ of $H-v_{1}$ by setting

$$
\varphi^{\prime}(u)= \begin{cases}c & \text { if } u=x, \\ \varphi(u) & \text { if } u \notin\left\{x, v_{1}\right\} .\end{cases}
$$

Clearly, $\varphi^{\prime}$ is a (proper) $L$-coloring of $H-v_{1}$ and since (b) does not hold for $x$ and $c, x \notin W_{j}^{\varphi^{\prime}}$, for $j=1, \ldots, l-1$. Indeed, it is not hard to see that

$$
W_{j}^{\varphi^{\prime}}=W_{j}^{\varphi}, \text { for } j=1, \ldots, l-1 .
$$

Moreover, since (b) does not hold for $x$ and $c, W_{l}^{\varphi^{\prime}}=W_{l}^{\varphi} \backslash\{x\}$, which together with (1) contradict that $\varphi \in \theta_{l}$. Hence, (a) or (b) holds for every vertex $u$ of $H-v_{1}$ and every color in $L(u) \backslash\{\varphi(u)\}$.

For a rank function $R$ defined as in part (iii) of Lemma 2.1, we say that $R$ is the rank function on $V(H)$ induced by $L$ and $\varphi$.

Let $F$ be a connected induced subgraph of a graph $G, v_{1}$ a fixed vertex of $F$ and $R: V(F) \rightarrow$ $\{1,2, \ldots,|V(F)|\}$ a rank function on the vertices of $F$. The triple $\left(F, v_{1}, R\right)$ is proper, if $R\left(v_{1}\right)=0$ and $R(u)>0$ for each vertex $u \in V(F) \backslash\left\{v_{1}\right\}$. We also say that $\left(F, v_{1}, R\right)$ is a proper triple of $G$. The next lemma gives an upper bound on the number of proper triples in a graph with bounded maximum degree.

Lemma 2.2. Let $G$ be a graph on $n$ vertices whose maximum degree is bounded by some absolute constant $\Delta$. The number of proper triples $\left(F, v_{1}, R\right)$, such that $F$ is a subgraph of $G$ with $m$ vertices does not exceed

$$
n \Delta^{m-1}(m-1) !(m-1)^{m-1} .
$$

Proof. The number of pairs $\left(F, v_{1}\right)$, where $F$ is a connected induced subgraph of $G$ on $m$ vertices, and $v_{1}$ is a fixed vertex of $F$, does not exceed

$$
n \Delta(2 \Delta)(3 \Delta) \ldots((m-1) \Delta)=n \Delta^{m-1}(m-1) !,
$$

because there are $n$ ways of selecting $v_{1}$, then we have $\Delta$ choices for the next vertex of $F$, and there are thereafter at most $(2 \Delta)$ choices for the next vertex, etc.

Now suppose that $F$ is a connected induced subgraph of $G$ and $v_{1}$ is a vertex of $F$. If $\left(F, v_{1}, R\right)$ is a proper triple, then $R\left(v_{1}\right)=0$ and $R(u)>0$ for each vertex $u \in V(F) \backslash\left\{v_{1}\right\}$. Since $F-v_{1}$ has $m-1$ vertices, there are at most $m-1$ ways of choosing $R(u)$ for a vertex $u \in V(F) \backslash\left\{v_{1}\right\}$ and thus the result follows.

Given a proper triple $\left(F, v_{1}, R\right)$ of a graph $G$ and a list assignment $L$ for $G$ such that $F$ is not $L$-colorable, we say that the quadruple $\left(F, v_{1}, R, L\right)$ is bad if there is an $L$-coloring $\varphi$ of $F-v_{1}$, such that $F, v_{1}, R, L$ and $\varphi$ satisfy conditions (i)-(iii) of Lemma 2.1 (with $F$ in place of $H$ ). In particular, $R$ is the rank function on $V(F)$ induced by $L$ and $\varphi$.

Consider a random $(k, \mathcal{C})$-list assignment for a graph $G$, where $\mathcal{C}=\{1,2, \ldots, \sigma\}$ and $k$ is a positive integer. The next lemma gives an upper bound on the probability that for a given proper triple $\left(F, v_{1}, R\right)$ of $G,\left(F, v_{1}, R, L\right)$ is bad. 
Lemma 2.3. Let $L$ be a random $(k, \mathcal{C})$-list assignment for a graph $G$ with maximum degree at most $\Delta$, where $\Delta$ is some positive integer. If $\left(F, v_{1}, R\right)$ is a proper triple of $G$ with $m=|V(F)|$, then

$$
\mathbb{P}\left[\left(F, v_{1}, R, L\right) \text { is bad }\right] \leq \frac{\sigma^{m-1}\left(\begin{array}{c}
\Delta \\
k
\end{array}\right)\left(\begin{array}{c}
\Delta k \\
k-1
\end{array}\right)^{m-1}}{\left(\begin{array}{l}
\sigma \\
k
\end{array}\right)^{m}} .
$$

Proof. Suppose that $\left(F, v_{1}, R\right)$ is a proper triple in $G$. To estimate the probability that $\left(F, v_{1}, R, L\right)$ is bad, we first count how many choices for a proper coloring $\varphi$ of $F-v_{1}$ we have. Then we count the number of list assignments $L$ for $F$ such that $\varphi$ is an $L$-coloring of $F-v_{1}$ and the conditions (i)-(iii) of Lemma 2.1 holds.

There are at most $\sigma^{m-1}$ ways of choosing a proper coloring $\varphi$ of $F-v_{1}$. Furthermore, since $F$ is not $L$-colorable it has maximum degree at least $k$, and thus $\Delta \geq k$. Note then that there are at most $\left(\begin{array}{l}\Delta \\ k\end{array}\right)$ ways of choosing the colors of the list $L\left(v_{1}\right)$ of $v_{1}$ after the coloring $\varphi$ has been chosen.

The rank function $R$ induces a partition $\left\{U_{0}, U_{1}, \ldots, U_{d}\right\}$ of $V(F)$, where $U_{i}$ consists of the vertices $w$ for which a shortest $(\varphi, L)$-alternating path from $v_{1}$ to $w$ has length $i$. Consider a vertex $w$ in $U_{1}$. We have already chosen a color $\varphi(w)$ for this vertex. Lemma 2.1 (iii) implies that we can include a color $c$ in $L(w)$ if there is a neighbor of $w$ in $F$ colored $c$ or a vertex $u \in N_{F}(w)$, such that $c \in L(u)$ and $R(u)<R(w)$. It follows that we have at most $\left(\begin{array}{c}\Delta k \\ k-1\end{array}\right)$ choices for the rest of the colors of $L(w)$. This holds for all the lists of vertices in $U_{1}$. Once we have chosen the colors for the vertices in $U_{1}$, we proceed similarly with the lists for vertices in $U_{2}$. If we include a color $c$ in a list $L(x)$ of a vertex $x \in U_{2}$, then either there is a neighbor of $x$ colored $c$, or there is a vertex $y \in N_{F}(x)$ such that $y \in U_{0} \cup U_{1}$ and $c \in L(y)$. It follows that there are at most $\left(\begin{array}{c}\Delta k \\ k-1\end{array}\right)$ ways of choosing the colors for $L(x) \backslash\{\varphi(x)\}$.

Proceeding similarly for all vertices of $F-v_{1}$ we may conclude that the remaining colors for the lists of the vertices of $F-v_{1}$ can be chosen in at most $\left(\begin{array}{c}\Delta k \\ k-1\end{array}\right)^{m-1}$ ways after that $\varphi$ has been fixed. Hence, the probability that $\left(F, v_{1}, R, L\right)$ is bad is at most

$$
\frac{\sigma^{m-1}\left(\begin{array}{c}
\Delta \\
k
\end{array}\right)\left(\begin{array}{c}
\Delta k \\
k-1
\end{array}\right)^{m-1}}{\left(\begin{array}{l}
\sigma \\
k
\end{array}\right)^{m}} .
$$

We are now in position to prove Theorem 1.3. Let $G=G(n)$ be a graph on $n$ vertices with maximum degree bounded by some positive integer $\Delta, k \geq 3$ a fixed positive integer, and let $L$ be a random $(k, \mathcal{C})$-list assignment for $G$.

Proof of Theorem 1.3. We will show that with high probability $G$ has no connected induced $L$ critical subgraph. This will imply the theorem.

By Lemma 2.1, it suffices to prove that if $\sigma(n)=\omega\left(n^{1 / k^{2}}\right)$, then with high probability $G$ does not contain a bad quadruple $\left(F, v_{1}, R, L\right)$. We will use easy first moment calculations.

If $\left(F, v_{1}, R, L\right)$ is bad, then $F$ has at least $k+1$ vertices. We first show that if $\left(F, v_{1}, R, L\right)$ is bad, then with high probability $F$ contains at most $\Delta^{k^{2}}$ vertices. Consider a path $P$ on $\nu$ vertices in $G$ with origin at some vertex $v$. The probability that there is an $L$-coloring $\varphi$ of $P-v$, such that $P$ is $(\varphi, L)$-alternating is at most

$$
\frac{\sigma(\sigma-1)^{\nu-2}\left(\begin{array}{l}
\sigma-1 \\
k-1
\end{array}\right)^{2}\left(\begin{array}{l}
\sigma-2 \\
k-2
\end{array}\right)^{\nu-2}}{\left(\begin{array}{l}
\sigma \\
k
\end{array}\right)^{\nu}} \leq \frac{k^{2 \nu}}{\sigma^{\nu-1}},
$$

because there are at most $\sigma(\sigma-1)^{\nu-2}$ ways of choosing the proper coloring $\varphi$ and thereafter at most $\left(\begin{array}{c}\sigma-1 \\ k-1\end{array}\right)^{2}\left(\begin{array}{c}\sigma-2 \\ k-2\end{array}\right)^{\nu-2}$ ways of choosing the list assignment $L$ for $P$ so that $\varphi$ is an $L$-coloring of $P-v$ 
and $P$ is $(\varphi, L)$-alternating. Moreover, the number of distinct paths in $G$ on $\nu$ vertices is at most $n \Delta^{\nu-1}$. Therefore, the expected number of paths $P$ in $G$ on at least $k^{2}+1$ vertices, for which there is an $L$-coloring $\varphi$ of $P-v$ such that $P$ is $(\varphi, L)$-alternating is at most

$$
\sum_{\nu=k^{2}+1}^{n} \frac{n \Delta^{\nu-1} k^{2 \nu}}{\sigma^{\nu-1}}=o(1),
$$

since $\sigma(n)=\omega\left(n^{1 / k^{2}}\right)$. Hence, by Markov's inequality, with high probability there is no $L$-coloring $\varphi$ of a subgraph of $G$ such that $G$ contains a $(\varphi, L)$-alternating path of length $k^{2}+1$.

Now, by Lemma 2.1, if $F$ is a subgraph of $G$ that belongs to a bad quadruple $\left(F, v_{1}, R, L\right)$, then there is an $L$-coloring $\varphi$ of $F-v_{1}$ such that all vertices of $F$ lie on $(\varphi, L)$-alternating paths with origin at $v_{1}$. Since with high probability the maximum length of such a path in $G$ is at most $k^{2}$, the maximum number of vertices in a subgraph of $G$ that is in a bad quadruple is with high probability at most

$$
1+\Delta+\Delta^{2}+\cdots+\Delta^{k^{2}-1} \leq \Delta^{k^{2}} .
$$

Let $X_{m}$ be a random variable counting the number of bad quadruples $\left(F, v_{1}, R, L\right)$ in $G$ such that $F$ has $m$ vertices and set

$$
X=\sum_{m=k+1}^{\Delta^{k^{2}}} X_{m} .
$$

Lemma 2.3 gives an upper bound on the probability that a given proper triple of $G$ on $m$ vertices is part of a bad quadruple. Additionally, by Lemma 2.2,

$$
f(m) \leq n \Delta^{m-1}(m-1) !(m-1)^{m-1},
$$

where $f(m)$ is the number of proper triples $\left(F, v_{1}, R\right)$ in $G$ such that $F$ has $m$ vertices. Let $p_{m}$ be the least number such that

$$
\mathbb{P}\left[\left(F, v_{1}, R, L\right) \text { is bad }\right] \leq p_{m},
$$

whenever $\left(F, v_{1}, R\right)$ is a proper triple in $G$ and $F$ has $m$ vertices. Since such a subgraph $F$ with high probability has at most $\Delta^{k^{2}}$ vertices if $\left(F, v_{1}, R, L\right)$ is bad, we conclude from Lemmas 2.2 and 2.3 that

$$
\begin{aligned}
\mathbb{P}[G \text { contains a bad quadruple }] & \leq \mathbb{E}[X]+o(1) \leq \sum_{m=k+1}^{\Delta^{k^{2}}} f(m) p_{m}+o(1) \\
& \leq \sum_{m=k+1}^{\Delta^{k^{2}}} n(\Delta m)^{m-1}(m-1) ! \frac{\sigma^{m-1}\left(\begin{array}{c}
\Delta \\
k
\end{array}\right)\left(\begin{array}{c}
\Delta k \\
k-1
\end{array}\right)^{m-1}}{\left(\begin{array}{c}
\sigma \\
k
\end{array}\right)^{m}}+o(1) \\
& =O\left(\frac{n}{\sigma^{(k-1)(k+1)+1}}\right) \sum_{m=0}^{\Delta^{k^{2}}}(m-1) !\left(\frac{k^{k} \Delta^{k} m}{\sigma^{k-1}}\right)^{m}=o(1),
\end{aligned}
$$

provided that $\sigma(n)=\omega\left(n^{1 / k^{2}}\right)$. 


\section{Graphs with girth greater than 3}

As mentioned before, Theorem 1.3 is best possible for general graphs with bounded maximum degree. Consider a graph $G=G(n)$ on $n$ vertices with bounded maximum degree and $\Theta(n)(k+1)$ cliques, where $k$ is a fixed integer satisfying $k \geq 2$. Let $L$ be a random $(k, \mathcal{C})$-list assignment for $G$, where $\mathcal{C}=\{1,2, \ldots, \sigma\}$. For such a graph, the threshold for the property of being $L$-colorable coincides with the threshold for disappearence of $(k+1)$-cliques where each vertex has the same list; that is, when $\sigma(n)=\omega\left(n^{1 / k^{2}}\right)$, then with high probability $G$ has no such cliques, and when $\sigma(n)=o\left(n^{1 / k^{2}}\right)$, then with high probability $G$ contains a $(k+1)$-clique where the vertices have identical lists. Hence, we might expect that if $H=H(n)$ is a triangle-free graph (and thus has no $(k+1)$-cliques if $k \geq 2)$ on $n$ vertices with bounded maximum degree and $L$ is a random $(k, \mathcal{C})$-list assignment for $H$, then we can find a better bound on $\sigma(n)$ and still guarantee that with high probability $H$ is $L$-colorable. As we will see, this is indeed the case.

We will proceed as in the proof of Theorem 1.3 and use first moment calculations to show that if $L$ is a random $(k, \mathcal{C})$-list assignment for $H$ and $\sigma(n)$ is large enough, then with high probability $H$ has no connected induced $L$-critical subgraph and thus is with high probability $L$-colorable. We need the following theorem.

Theorem 3.1. [1] For $g \geq 3$ and $\delta \geq 3$ put

$$
n_{0}(g, \delta)= \begin{cases}1+\frac{\delta}{\delta-2}\left((\delta-1)^{(g-1) / 2}-1\right) & \text { if } g \text { is odd, } \\ \frac{2}{\delta-2}\left((\delta-1)^{g / 2}-1\right) & \text { if } g \text { is even } .\end{cases}
$$

Then a graph $G$ with minimum degree $\delta$ and girth $g$ has at least $n_{0}(g, \delta)$ vertices.

The case when $k=2$ was dealt with in [4], so assume that $k$ is a fixed integer satisfying $k>2$, $g \geq 4$ is a fixed integer, $H=H(n)$ is a graph on $n$ vertices, the maximum degree of $H$ is bounded from above by a positive integer $\Delta$, and $H$ has girth at least $g$. Suppose that

$$
\sigma(n)=\omega\left(n^{\frac{1}{(k-1) n_{0}(g, k)+1}}\right),
$$

where $n_{0}$ is defined as in Theorem 3.1, and let $L$ be a random $(k, \mathcal{C})$-list assignment for $H$. If $H$ contains a connected induced $L$-critical subgraph $F$, then $F$ has minimum degree at least $k$. Since $F$ has girth at least $g$, Theorem 3.1 implies that $F$ has at least $n_{0}(g, k)$ vertices. We set $Q(k)=n_{0}(g, k)$, and by Theorem 3.1 , we have

$$
Q(k)= \begin{cases}1+k\left(1+(k-1)+(k-1)^{2}+\cdots+(k-1)^{\frac{g-3}{2}}\right), & \text { if } g \text { is odd } \\ 2\left(1+(k-1)+(k-1)^{2}+\cdots+(k-1)^{\frac{g-2}{2}}\right), & \text { if } g \text { is even }\end{cases}
$$

Moreover, by proceeding as in the proof of Theorem 1.3, it is not hard to show that if $F$ is $L$-critical and thus by Lemma 2.1 belongs to a bad quadruple $(F, v, R, L)$ of $H$, then with high probability $F$ contains at most $r(\Delta, g, k)$ vertices, where $r(\Delta, g, k)$ is a function that only depends on $\Delta, g$ and $k$. (In particular, $r(\Delta, g, k)$ does not depend on $n$.) Hence, by Lemma 2.1, it suffices to prove that with high probability $H$ contains no bad quadruple $(F, v, R, L)$ such that $Q(k) \leq|V(F)| \leq r(\Delta, g, k)$.

Let $Z_{m}$ be a random variable counting the number of bad quadruples $(F, v, R, L)$ in $H$ such that $F$ has $m$ vertices, and set

$$
Z=\sum_{m=Q(k)}^{r(\Delta, g, k)} Z_{m}
$$


Using Lemmas 2.2 and 2.3 we may now conclude that

$$
\begin{aligned}
\mathbb{P}[H \text { contains a bad quadruple }] & \leq \mathbb{E}[Z]+o(1) \\
& \leq \sum_{m=Q(k)}^{r(\Delta, g, k)} n(\Delta m)^{m-1}(m-1) ! \frac{\sigma^{m-1}\left(\begin{array}{c}
\Delta \\
k
\end{array}\right)\left(\begin{array}{c}
\Delta k \\
k-1
\end{array}\right)^{m-1}}{\left(\begin{array}{c}
\sigma \\
k
\end{array}\right)^{m}}+o(1) \\
& =O\left(\frac{n}{\sigma^{(k-1) Q(k)+1}}\right) \sum_{m=0}^{r(\Delta, g, k)}(m-1) !\left(\frac{k^{k} \Delta^{k} m}{\sigma^{k-1}}\right)^{m}=o(1),
\end{aligned}
$$

provided that $\sigma(n)=\omega\left(n^{\frac{1}{(k-1) Q(k)+1}}\right)$.

Let us determine $(k-1) Q(k)+1$ explicitly for some small values of $g$. When $g=4$, then we have to require that $\sigma(n)=\omega\left(n^{1 /\left(2 k^{2}-2 k+1\right)}\right)$ to ensure that there is an $L$-coloring of $H$ with high probability, and when $g=5$, then it suffices to require that $\sigma(n)=\omega\left(n^{1 /\left(k^{3}-k^{2}+k\right)}\right)$. We collect these results in the following proposition.

Proposition 3.2. Let $G=G(n)$ be a graph on $n$ vertices with bounded maximum degree and fixed girth $g$. Suppose that $k$ is a fixed integer satisfying $k \geq 3$ and that $L$ is a random $(k, \mathcal{C})$-list assignment for $G$.

(i) If $g=4$ and $\sigma(n)=\omega\left(n^{1 /\left(2 k^{2}-2 k+1\right)}\right)$, then with high probability $G$ has an L-coloring.

(ii) If $g=5$ and $\sigma(n)=\omega\left(n^{1 /\left(k^{3}-k^{2}+k\right)}\right)$, then with high probablity $G$ has an L-coloring.

(iii) If $g>5$, then there is a polynomial $P(k)$ in $k$ of degree $\lceil g / 2\rceil$, such that if $\sigma(n)=\omega\left(n^{1 / P(k)}\right)$, then with high probability $G$ is L-colorable. Moreover,

$$
P(k)=(k-1) Q(k)+1,
$$

where $Q(k)$ is given by $(2)$.

Remark. Proposition 3.2 is not best possible. Consider, for example, the simplest case when $g=4$. The lower bound $\sigma(n)=\omega\left(n^{1 /\left(2 k^{2}-2 k+1\right)}\right)$ comes from the fact that a graph with minimum degree $k$ and girth 4 contains at least $2 k$ vertices. If $k=3$, then the unique minimal graph with girth 4 and minimum degree 3 is the complete bipartite graph $K_{3,3}$. Thus $K_{3,3}$ is the smallest graph which might be in a bad quadruple in $G$. However, $K_{3,3}$ is easily verified to be 3 -choosable, which means that it cannot be in a bad quadruple in $G$. Hence, the smallest graph which might be in a bad quadruple in $G$ has at least 7 vertices and consequently: for the case when $k=3$ and $g=4$ it is possible to establish a better lower bound on $\sigma$ in Proposition 3.2. In general, to get better bounds in Proposition 3.2, we would need to know how many vertices the smallest non- $k$-choosable graph with girth $g$ has.

\section{Complete graphs}

In this section we prove Theorem 1.4. Recall that $\mathcal{C}=\{1,2, \ldots, \sigma(n)\}$. Note that in general, if $L$ is a list assignment for $K_{n}$ with colors from $\mathcal{C}$ and $K_{n}$ is $L$-colorable, then $\sigma(n) \geq n$.

Consider a random $(k, \mathcal{C})$-list assignment $L$ for $K_{n}$, where $k \geq 3$ is a fixed integer. In order to prove Theorem 1.4 we will use the standard uniform probability space of $k$-uniform random 
hypergraphs $\mathcal{H}_{k}(n, m)$. We will also need to consider the space $\mathcal{H}_{k}^{*}(n, m)$ consisting of all $k$-uniform multihypergraphs on $n$ labelled vertices and $m$ edges and where each such multihypergraph is equally likely.

The following reformulation of the original problem will prove useful: Let $L$ be a $k$-list assignment for $K_{n}$, where the colors of each list are chosen from $\mathcal{C}$. Define the hypergraph $T=T(L)$ by letting $\mathcal{C}$ be its vertex set and include the edge $\left\{c_{1}, \ldots, c_{k}\right\}$ in $E(T)$ if and only if $L(v)=\left\{c_{1}, \ldots, c_{k}\right\}$ for some vertex $v$ of $K_{n}$ (multiple edges allowed). If $L$ is a random $(k, \mathcal{C})$-list assignment, that is, the color lists are chosen uniformly at random from all $k$-subsets of $\mathcal{C}$, then clearly $T$ is a random hypergraph distributed as $\mathcal{H}_{k}^{*}(\sigma, n)$. However, the expected number of pairs of multiple edges in $T$ is

$$
\left(\begin{array}{l}
\sigma \\
k
\end{array}\right)\left(\begin{array}{c}
\left(\begin{array}{c} 
\\
k
\end{array}\right)+n-3 \\
n-2
\end{array}\right)\left(\begin{array}{c}
\left(\begin{array}{c}
\sigma \\
k
\end{array}\right)+n-1 \\
n
\end{array}\right)^{-1}=o(1),
$$

if $k \geq 3$ and $\sigma=\Theta(n)$. Thus, by Markov's inequality we may simply ignore multiple edges and consider $T$ distributed as $\mathcal{H}_{k}(\sigma, n)$.

We will now show that $K_{n}$ has an $L$-coloring if and only if there is an injective mapping $f: E(T) \rightarrow V(T)$, such that if $f(e)=v$ then $e$ contains $v$, for all edges $e$ and vertices $v$ in $T$. If $f$ satisfies this condition and is injective then $f$ is allowed.

Given an allowed map $f: E(T) \rightarrow V(T)$, we simply color the vertex $u \in V\left(K_{n}\right)$ with the color $f(e)$, where $e$ is the edge of $T$ corresponding to $L(u)$. Since $f$ is injective, this will result in a (proper) $L$-coloring of $K_{n}$. Conversely, suppose that $\varphi$ is an $L$-coloring of $K_{n}$. Then $\varphi$ defines an allowed map $f: E(T) \rightarrow V(T)$ in the following way: suppose that $v \in V\left(K_{n}\right)$ with $L(v)=\left\{c_{1}, \ldots, c_{k}\right\}$ and $\varphi(v)=c_{1}$. Then $e=\left\{c_{1}, \ldots, c_{k}\right\}$ is the edge in $T$ corresponding to $L(v)$ and we set $f(e)=c_{1}$. Since $\varphi$ is a proper coloring of $V\left(K_{n}\right)$, we obtain an allowed map $f: E(T) \rightarrow V(T)$ by repeating this for every vertex of $K_{n}$.

Now consider a bipartite graph $B$ with parts $V=V(T)$ and $E=E(T)$ and where $v \in V$ and $e \in E$ are adjacent if $e$ contains $v$ in $T$. An allowed map $f: E \rightarrow V$ corresponds to a matching in $B$ that saturates every vertex of $E .^{2}$ By Hall's condition, such a matching exists unless there is a set $S \subseteq E$ such that $\left|N_{B}(S)\right|<|S|$, where $N_{B}(S)$ is the set of vertices in $B$ that are adjacent to some vertex in $S$. Suppose that there is such a set in $B$ and let $S$ be a minimal such set. It is not hard to show that then $|S|=\left|N_{B}(S)\right|+1$, every vertex in $N_{B}(S)$ is adjacent to at least two vertices in $S$, and $B\left[S \cup N_{B}(S)\right]$ is connected. In $T, B\left[S \cup N_{B}(S)\right]$ corresponds to a connected subhypergraph with $|S|-1$ vertices, $|S|$ edges and minimum degree at least 2 (in the subhypergraph). We call such a subhypergraph bad.

By the above reformulation, to prove Theorem 1.4 it suffices to show that if $T$ is a random hypergraph distributed as $\mathcal{H}_{3}(n, m)$, then there is a number $s^{*} \approx 1.222$ such that for any $s>s^{*}$, if $s m \leq n$, then with high probability $T$ does not have a bad subhypergraph. To obtain as optimal value of $s^{*}$ as possible we would need a good upper bound on the number of connected hypergraphs $F$ with minimum degree $2, t$ vertices and $t+1$ edges when $t=\Theta(n)$. (It is well-known, see e.g. [9], that if $T$ is distributed as $\mathcal{H}_{3}(n, m)$ and $m=\Theta(n)$, then with high probability every induced subhypergraph of $T$ of order $o(n)$ has average degree less than 3.) However, as finding such a useful upper bound seems difficult, we will use the following theorem, which is easily seen to follow from Theorem 1 in [9]. An $r$-core in a hypergraph is a non-empty connected maximal subhypergraph of minimum degree at least $r$. Obviously, if $T$ has a bad subhypergraph, then it has a non-empty 2-core. (Theorem 1 in [9] was originally proved for the model $\mathcal{H}_{k}(n, p)$, where each of the $\left(\begin{array}{l}n \\ k\end{array}\right)$ potential edges is present with probability $p$, independently of all other potential edges. Since the

${ }^{2}$ The equivalence of list coloring of a complete graph to finding a matching in a bipartite graph was first proved by Vizing [10]. 
property of having $r$-cores is monotone, the corresponding result also holds for the model $\mathcal{H}_{k}(n, m)$ when $m=\left(\begin{array}{l}n \\ k\end{array}\right) p$. For details, see e.g. [6].)

Theorem 4.1. [9] Let $k \geq 3$. Define

$$
c^{*}=\min _{x>0} \frac{x(k-1) !}{\left(1-e^{-x}\right)^{k-1}},
$$

set $s^{*}=\frac{k !}{c^{*}}$ and let $H$ be a random hypergraph distributed as $\mathcal{H}_{k}(n, m)$. For any $\epsilon>0$

(i) if $m \leq(1-\epsilon) \frac{n}{s^{*}}$, then with high probability $H$ has no 2-core,

(ii) if $m \geq(1+\epsilon) \frac{n}{s^{*}}$, then with high probability $H$ has a 2-core.

It is easy to verify that if $k=3$, then Theorem 4.1 yields that $c^{*} \approx 4.9108$ and thus $s^{*} \approx 1.222$. This concludes the proof of Theorem 1.4.

\section{Acknowledgements}

The author thanks Jonas Hägglund for helping him with some computer simulations at an early stage of the research.

The author also thanks the referees for helpful suggestions which improved the presentation of the paper.

\section{References}

[1] B. Bollobás, Modern Graph Theory, Springer, New York, 1998.

[2] C. J. Casselgren, On some graph coloring problems, Doctoral thesis, Umeå University, 2011.

[3] C. J. Casselgren, Vertex coloring complete multipartite graphs from random lists of size 2, Discrete Mathematics 311 (2010), pp. 1150-1157.

[4] C. J. Casselgren, Coloring graphs from random lists of size 2, European Journal of Combinatorics 33 (2012), 168-181.

[5] P. Erdős, A. L. Rubin, H. Taylor, Choosability in graphs, Proceedings West Coast Conf. on Combinatorics, Graph Theory and Computing, Congressus Numerantium XXVI, 1979, pp. $125-157$.

[6] S. Janson, T. Łuczak, A. Ruciński, Random Graphs, Wiley, New York, 2000.

[7] M. Krivelevich, A. Nachmias, Coloring powers of cycles from random lists, European Journal of Combinatorics 25 (2004), pp. 961-968.

[8] M. Krivelevich, A. Nachmias, Coloring Complete Bipartite Graphs from Random Lists, Random Structures and Algorithms 29 (2006), pp. 436-449.

[9] M. Molloy, Cores in random hypergraphs and boolean formulas, Random Structures and Algorithms 27 (2005), pp. 124-135.

[10] V. G. Vizing, Coloring the vertices of a graph with prescribed colors, Metody Diskretnogo Analiza Teorii Kodov i Skhem 29 (1976), pp. 3-10 (in Russian). 